\title{
Peri-operative Therapies for Localized Retroperitoneal Soft Tissue Sarcoma
}

\author{
Stefano Radaelli, MD, ${ }^{1}$ Anant Desai, MD, ${ }^{2}$ Chiara Colombo, MD, ${ }^{3}$ Marco Fiore, MD $^{3}$ and Alessandro Gronchi, MD ${ }^{4}$ \\ 1. Fellow Surgeon, Department of Surgery, National Cancer Institute, Milan; 2. Attending Surgeon, Department of Surgery, National Cancer Institute, Milan and \\ Queen Elizabeth Medical Centre, Birmingham; 3. Attending Surgeon, Department of Surgery, National Cancer Institute, Milan; 4. Chief, Sarcoma Service, \\ Department of Surgery, National Cancer Institute, Milan
}

\begin{abstract}
Retroperitoneal soft tissue sarcomas are rare solid tumors that usually arise in the retroperitoneum or the pelvis. They are often large at presentation and push adjacent structures, displacing them, anatomically. The most common adult histotypes are liposarcoma and leiomyosarcoma. Five-year overall survival is less than $50 \%$ and is mainly determined by the high incidence of local relapse and to a lesser extent by their capacity to metastasize. Radical surgery is still the most effective treatment. While an aggressive surgical policy consisting of multivisceral resections 'en bloc' with the mass has undoubtedly decreased, the rate of local recurrence, prognosis remains poor. Retrospective analyses and early prospective data suggest a benefit from using neoadjuvant radiotherapy in order to improve local control. Chemotherapy plays an important role in advanced disease, but very little evidence supports a real advantage in delivering it prior to surgery to make the resection more likely to achieve clear margins or postoperatively to decrease the risk of systemic recurrence. Further studies are required to explore the role of these neoadjuvant/adjuvant therapies, particularly in the context of new developments in molecular therapeutic agents.
\end{abstract}

\section{Keywords}

Sarcoma, retroperitoneal sarcoma, chemotherapy, surgery, radiotherapy, local control, survival

Disclosure: The authors have no conflicts of interest to declare.

Received: January 17, 2012 Accepted: January 31, 2012 Citation: Oncology \& Hematology Review, 2012;8(1):65-70 DOI: 10.17925/OHR.2012.08.1.65

Correspondence: Alessandro Gronchi, MD, Sarcoma Service, Department of Surgery, Fondazione IRCCS Istituto Nazionale Tumori, Via Venezian 1, 20133 Milan, Italy.

E: alessandro.gronchi@istitutotumori.mi.it

Retroperitoneal soft tissue sarcomas (RSTSs) are uncommon tumors composing less than $1 \%$ of all adult cancers. They account for approximately $15 \%$ of soft tissue sarcomas (STSS), i.e., the most frequent site of mesenchymal malignancy after the lower extremities. ${ }^{1}$ Although many different sarcoma histotypes exist, the most common in the retroperitoneum are liposarcoma (41\%), leiomyosarcoma (28\%), pleomorphic sarcoma, solitary fibrous tumor, synovial sarcoma, and malignant peripheral nerve sheath tumor. Other subtypes, such as myofibroblastic inflammatory tumor, low-grade fibromyxoid sarcoma, and myxofibrosarcoma, have to be considered rarities. About $50 \%$ of RSTSs are reported to be of high grade in terms of their biologic aggressiveness. ${ }^{2}$ The retroperitoneum can also host a wide spectrum of other benign tumors, such as paragangliomas, angiomyolipomas, and schwannomas as well as primary epithelial malignancies from the kidney and the adrenal gland. It is also a common site for lymphomas and metastatic nodal lesions from germ cell neoplasms.

The clinical presentation of an RSTS is that of a large mass in the right or left retroperitoneal space that is able to push and dislocate the surrounding viscera and vessels, without showing an infiltrative pattern of growth. Initially, these neoplasms are asymptomatic or give rise to few symptoms causing non-specific abdominal discomfort or pain, and diagnosis is often late. If found early, it is usually because the patient has had imaging for other indications and the mass is found incidentally. Over time, patients develop a palpable mass with a significant increase in volume and girth of the abdomen.

The diagnostic approach generally starts with a contrast-enhanced abdominal and thoracic computed tomography scan. This is usually enough to achieve complete oncologic staging. Positron emission tomography, magnetic resonance imaging, and bone scans are seldom required. A pre-operative percutaneous core biopsy, where possible, should be performed. The histologic diagnosis, together with the radiologic findings and the patient's performance status, should guide the multidisciplinary decision about the most appropriate therapeutic approach. An aggressive surgical policy, consisting of multiple visceral resection 'en bloc' with the mass, is still the most effective strategy to cure a primary retroperitoneal sarcoma. At the same time, a multidisciplinary approach, mostly based on pre-operative chemo-radiation therapy, is increasingly important in high-grade or unresectable RSTSS. ${ }^{3}$

\section{Surgery}

Surgery is the mainstay of treatment in localized disease. Local control is critical and largely depends on the quality of surgery. Anatomic constraints in the retroperitoneum limit the ability to achieve wide 
Table 1: Five-year Overall Survival and Local Recurrence Rates in the Most Representative Surgical Series of Retroperitoneal Soft Tissue Sarcoma

\begin{tabular}{|c|c|c|c|c|c|c|}
\hline Study & Study Period & No of Patients & $\begin{array}{l}\text { Median FU } \\
\text { (months) }\end{array}$ & $\begin{array}{l}\text { Complete } \\
\text { Resection (\%) }\end{array}$ & Five-year OS (\%) & Five-year LRFS (\%) \\
\hline Lewis et al., 2004 ${ }^{4}$ & 1982-1997 & 231 & 28 & 80 & 54 & 59 \\
\hline Stoeckle et al., $2001^{5}$ & 1980-1994 & 145 & 47 & 65 & 49 & 42 \\
\hline $\begin{array}{l}\text { Ferrario and } \\
\text { Karakousis, } 2003^{6}\end{array}$ & 1977-2001 & 79 & 41 & 99 & 65 & 43 \\
\hline Kilkenny et al., 19967 & 1970-1994 & 63 & NR & 78 & 48 & NR \\
\hline Hassan et al., $2004^{8}$ & 1983-1995 & 97 & 36 & 55 & 51 & 56 \\
\hline Van Dalen et al., $2007^{9}$ & 1989-1994 & 143 & 122 & 70 & 39 & NR \\
\hline Lehnert et al., $2009^{10}$ & 1998-2002 & 71 & 89 & 85 & 51 & 59 \\
\hline Strauss et al., $2010^{11}$ & 1990-2009 & 200 & 29 & 90 & 68 & 55 \\
\hline Gronchi et al., $2012^{12}$ & 2002-2008 & 191 & 48 & 94 & 67 & 72 \\
\hline
\end{tabular}

$F U=$ follow-up; $L R F S=$ local recurrence-free survival; $N R$ = not reported; OS = overall survival.

Figure 1: Aggressive Surgical Policy-Multivisceral Resection 'En Bloc' with the Retroperitoneal Sarcoma

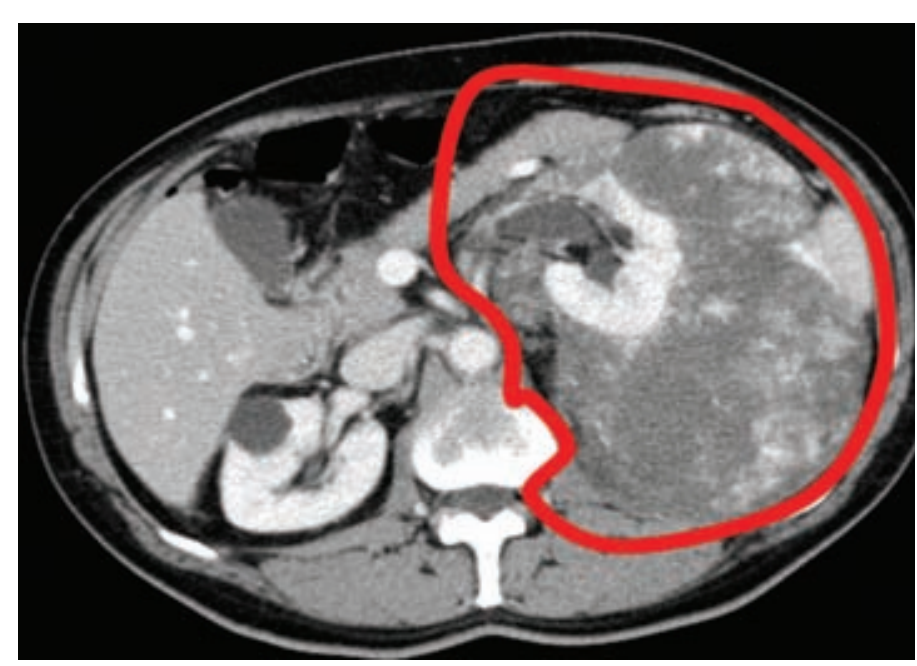

resection margins and surgery is marginal by definition. Consequently, local recurrence is much more frequent than at any other anatomic site and is the leading cause of death. Long-term survival following resection of an RSTS is in the range of 30-50 \% (see Table 1). ${ }^{4-12}$

Some authors have advocated the widest possible surgical resection at presentation, although currently this is not universally accepted. In contrast to extremity soft tissue sarcomas, no clear definitions of surgical adequacy are available and recommendations are limited to the achievement of gross complete resection, with the need to resect adjacent organs only when there is direct involvement by tumor.

Recent retrospective analyses favor a more liberal approach reporting a greater than $70 \%$ local control at five years. ${ }^{13,14}$ The surgical strategy consists of 'en bloc' resection of surrounding adjacent organs (i.e., loco-regional peritonectomy, resection of surrounding viscera, mainly kidney and colon, or psoas muscle if in close proximity to the tumor; see Figure 1). This more liberal policy was shown to be associated with a decrease in five-year local recurrence rates from 49-28 \%. ${ }^{13,14}$ This improvement was paralleled by an increase of five-year overall survival from $48-66 \%$.
The main problem in retroperitoneal sarcomas when compared with extremity sarcomas is that the surrounding normal tissues are more likely to be visceral structures rather than muscles. The dilemma is that the real concern about potential morbidity related to extended resections must be balanced against the probability of not achieving a negative pathologic resection margin.

Major morbidity of such an approach is reported to be close to $18 \%$, while the risk of mortality is around $3 \%$. These results are comparable to those following all other major abdominal operations. ${ }^{15,16}$

Surgery has a higher potential to cure low and intermediate grade tumors, since survival largely depends on local control. It seems rational that in tumors characterized by a less aggressive biology, more aggressive surgical strategies that achieve better short- to medium-term local control, will improve the ultimate prognosis but long-term follow-up is needed to confirm this. Among the different histologic subtypes, well- and de-differentiated liposarcomas do not usually develop distant metastases and appear those most likely to derive the greatest benefit from extended resections. Similar considerations could apply to solitary fibrous tumors, as well as for other rarer low-grade sarcomas. In contrast, high-grade tumors, such as leiomyosarcoma, have a more aggressive biology characterized by a higher tendency to metastasize. Therefore, local treatment should be complemented by systemic therapy.

In recognition of the fact that these tumors tend to recur, there has been increasing interest in the past decade in a multidisciplinary approach in order to decrease RSTS local relapse and improve long-term survival. 2,17,18

\section{Radiotherapy}

Several prospective, randomized studies have demonstrated the effectiveness of post-operative radiotherapy (RT) in improving local control in limb and trunk STS. ${ }^{19,20}$ Based on these results, many investigators have combined RT and surgery in RSTSs in order to achieve the kinds of outcomes seen in extremity sarcomas.

The complexity of delivering radiation therapy in RSTSS is mostly due to the large volume that requires treatment and the proximity to critical uninvolved structures such as the bowel, kidney, uterus, liver, spinal 
cord, and peripheral nerves. Beside the rarity of the disease, these factors represent the most important reasons for the scarcity of published clinical trials or prospective studies regarding the use of radiation therapy in RSTSS. ${ }^{2,15,17}$ This is clearly exemplified by the first randomized trial (29031) led by the American College of Surgeons Oncology Group that would have compared pre- and post-operative RT in RSTSS, but was stopped due a lack of patient recruitment. ${ }^{2}$

To date, only one randomized controlled trial, performed between 1980 and 1985 at the US National Cancer Institute is available. Thirty-five patients were recruited and all patients underwent macroscopically complete resection. The patients were divided into two groups with the first group of 20 patients receiving adjuvant external beam radiotherapy (EBRT) (35-40 Gy to an extended field and 15-20 Gy to a boost field) and a randomly assigned second group of 15 patients also treated with post-operative EBRT (35-40 Gy) but also intravenous misonidazole (a radiosensitizer) and intra-operative radiotherapy (IORT) (20 Gy). Local recurrence was higher in the EBRT group (80 versus $40 \%$ in the EBRT plus IORT group). Treatment-related toxicity, mostly radiation enteritis and neuropathy, was more common in the EBRT plus IORT group. ${ }^{2}$

A retrospective French study delivering adjuvant RT in 60 patients with RSTS documented a five-year local relapse-free survival rate of 55 against $23 \%$ in 34 patients who did not receive post-operative RT. ${ }^{5}$ Another retrospective analysis of 261 patients that received adjuvant RT and surgery documented a risk of local relapse at five years of $31 \%$ compared with $36 \%$ in patients that had surgery alone. ${ }^{21}$

Catton et al. in a retrospective analysis regarding prognostic factors in RSTSs demonstrating that post-operative EBRT in doses >35 Gy, after complete surgery, delayed local recurrence..$^{22}$ The conclusions were that administrating RT postoperatively improved local control but at the expense of increased radiation-induced complications. ${ }^{23}$ Paumier et al. have shown, however, that it is possible to reduce the degree of radiation-related toxicity by using intensity-modulated RT (IMRT) instead of traditional EBRT in the post-operative setting. They conducted a prospective study recruiting 14 patients who had undergone complete surgical resection and administered adjuvant IMRT. The study showed mild acute toxicity, but longer follow-up is needed to evaluate late effects, particularly damage to the bowel and kidneys, and radiation-induced cancers. Intensity-modulated RT provides the opportunity to treat wide areas in the retroperitoneum that were previously impossible. ${ }^{24}$

In an attempt to reduce complications due to post-operative RT, many sarcoma centers have tried a neo-adjuvant approach. This strategy is attractive for a number of reasons. First, irradiating the tumor may reduce its size and possibly decrease the number of organs that require resection with the tumor. Second, the field to be treated can be defined more accurately due to the presence of the tumor-the large mass present in the retroperitoneum displaces adjacent viscera and limits their RT-related toxicity. Finally, the tumor pseudocapsule may thicken when treated with RT, making surgical dissection easier and minimizing the risk of local relapse or peritoneal seeding (see Figure 2). ${ }^{1,2,18}$

The advantage of delivering radiation therapy preoperatively was first described when data were published from two combined single arms

\section{Figure 2: Planning of Pre-operative
Intensity-modulated Radiotherapy}

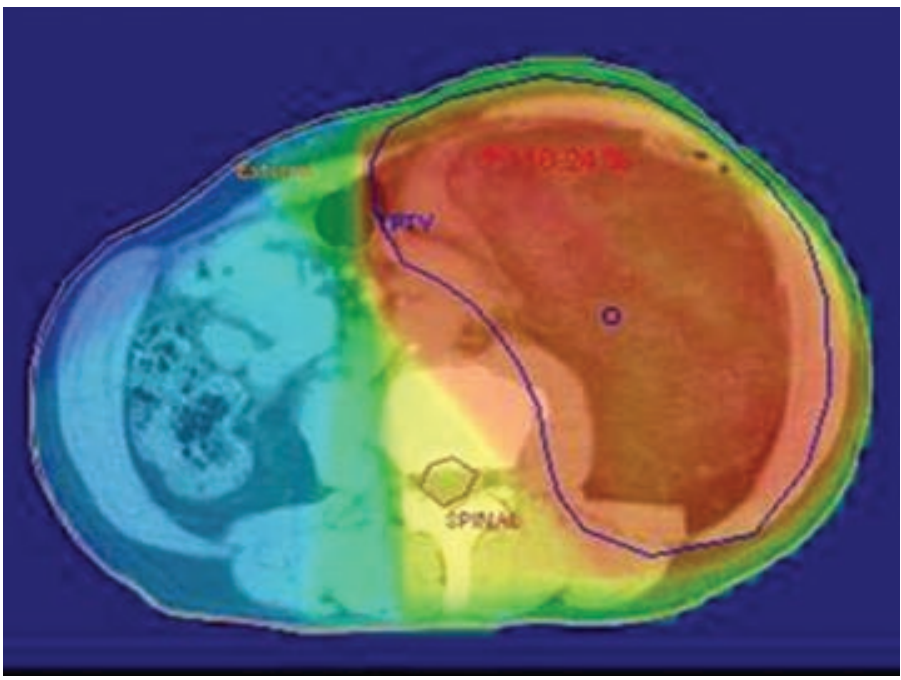

Pre-operative intensity-modulated radiotherapy (IMRT): an advanced mode of high-precision radiotherapy that utilizes computer-controlled linear accelerators to deliver precise radiation doses to a malignant tumor or specific areas within the tumor. IMRT allows the dose to conform more precisely to the $3 \mathrm{D}$ shape of the tumor by modulating or controlling the intensity of the radiation beam in multiple small volumes. IMRT also allows higher radiation doses to be focused to regions within the tumor while minimizing the dose to surrounding normal critical structures.

trials that explored the use of adjuvant EBRT in RSTSs. ${ }^{25}$ Seventy-two patients were treated with pre-operative EBRT and complete surgical resection. Patients enrolled at the University of Toronto also received post-operative brachytherapy, while patients treated at the University of Texas received IORT and some received chemotherapy concurrent with pre-operative RT.

The majority of patients (89\%) completed the planned pre-operative RT and most (79 \%) underwent surgery with a curative intent, with $95 \%$ going on to have a radical resection. Five-years' local recurrence and disease-free survival were 60 and $61 \%$ respectively. These survival rates compare favorably with historic data from patients with retroperitoneal sarcomas that were treated with surgery alone. Relatively low toxicity was reported for EBRT. Brachytherapy, however, was associated with major complications and treatment was interrupted in a number of cases. Concurrent chemotherapy required hospitalization in a small proportion (11\%) of patients and was generally well tolerated. ${ }^{2,21-25}$

Taken together, all these findings support the use of RT in the pre-operative setting. Further studies are needed to better investigate its use.

\section{Chemotherapy}

No trial addressing the specific role of chemotherapy in RSTSs has been ever conducted. Most of the available ones included all sites, the retroperitoneum being one of the less represented. This may be a potential limitation in extending the reported results to the retroperitoneum.

Fourteen randomized clinical trials, all comparing doxorubicin-based adjuvant chemotherapy regimens with no-treatment post-surgery, were pooled in a meta-analysis and published in 1997. This study, including a total of 1,568 patients, summarizes the evidence that emerged from the 
first-generation clinical trials employing doxorubicin-based protocols as an adjuvant in primary STS. ${ }^{26}$ Its conclusions indicate that adjuvant chemotherapy, as given in these early trials, may provide an absolute relapse-free advantage in the order of $10 \%$, and a lower overall survival improvement, which in fact did not reach statistical significance despite the large sample size. Of interest, some degree of delay in relapse as well as a lower local failure rate was observed in several trials. The limitations of these trials do not lie only in the chemotherapy regimen as patient selection was also important. Taken overall, these trials included STS at all sites, from the extremities to the uterus, all grades of malignancy, and all histologic subtypes (including a number of gastrointestinal stromal tumors, rendering adjuvant chemotherapy apparently less active in leiomyosarcoma). Clearly, the larger the trial, the more heterogeneous the patient population. In addition, the largest trials were performed by a high number of participating institutions, with possible inequalities in the quality of care, given the rarity of STS. In other words, in a rare, highly heterogeneous disease, the statistical precision tends to correlate directly with the homogeneity of patient populations and, possibly, quality of care. Consequently, a benefit in favor of adjuvant chemotherapy was seen mainly in the smallest trials, and one may view this with suspicion as possibly the result of publication bias. From the clinical point of view, however, it is equally true that the largest trials were also more heterogeneous and therefore less likely to allow detection of a benefit, if any.

An improvement in overall survival has been demonstrated in a single randomized trial, which involved an anthracycline (epirubicin) plus ifosfamide, although the trial had relatively short follow-up. ${ }^{27}$ This overall survival benefit was confirmed in a recent meta-analysis ${ }^{28}$ which also included three other recent trials of anthracycline plus ifosfamide, ${ }^{27,29,30}$ although it excluded a major, negative, unpublished trial. ${ }^{31}$ The survival benefit in the meta-analysis was limited but statistically significant (hazard ratio $0.77, p=0.01$ ). This advantage has been also suggested in the recent Italian Sarcoma Group trial, comparing three versus five cycles of full-dose chemotherapy (combination of anthracycline and ifosfamide) in high-risk extremity and trunk wall STS. No difference was observed between the two arms but a better overall outcome was observed when compared to the nomogram prediction for the same population..$^{32}$ These data suggest that a more prolonged neoadjuvant chemotherapy treatment does not improve outcome and may be associated with increased toxicity.

Furthermore, some retrospective analyses have been published, in an attempt to address this issue from a different angle, given the difficulty in carrying out prospective randomized trials in such a rare disease. The largest number of patients (674) was reached by a study providing a retrospective analysis of STS patients treated from 1984-1999 in two referral sarcoma centers in the US, the Memorial Sloan-Kettering Cancer Center (MSKCC) and MD Anderson Cancer Center. ${ }^{33}$ The policy in these institutions was different enough to result in 38 and $66 \%$ of patients being treated with adjuvant chemotherapy, respectively. By combining the two series, a comparison was made between those treated with adjuvant chemotherapy and those who were not, apparently having equivalent prognostic measures. The relapse-free and the overall disease-specific survival were almost the same in the control and treatment groups, although with some delay in tumor relapse for patients treated with chemotherapy. Clearly, these results are suggestive of a lack of benefit for adjuvant chemotherapy. Aside from potential biases in these comparisons, in $88 \%$ of cases no further analysis was made with regard to the chemotherapy regimen, which was doxorubicin alone or in combination with other drugs (including ifosfamide). Furthermore, the distant relapse-free rate in this patient population was 50-60\%. Hence this analysis does not encourage the use of adjuvant chemotherapy, but neither does it amount to definitive evidence disproving any possible role for a full-dose combination chemotherapy in some high-risk subsets of STS patients.

Another retrospective analysis combined the MSKCC and the University of California Los Angeles series of patients with large $(>5 \mathrm{~cm})$, high-grade, extremity liposarcomas. ${ }^{34} \mathrm{~A}$ benefit was seen, in terms of both disease-specific survival and distant recurrence-free survival, for patients treated with adjuvant/neoadjuvant Ifosfamide plus doxorubicin but not for those treated with doxorubicin alone, when compared with those who were not treated with adjuvant chemotherapy. This result highlights the potential value of studying a single histologic type, and possibly the importance of the chemotherapy regimen (whether ifosfamide has greater activity in liposarcoma or whether the combination of drugs is important is unclear). Likewise, in synovial sarcoma, a retrospective comparison was made between a pediatric and an adult patient population within the same institution, which followed a different policy for the two groups. ${ }^{35}$ Some advantage in favor of adjuvant chemotherapy is suggested by the data, especially for patients with tumors $>5 \mathrm{~cm}$, in what is considered to be an aggressive histologic type.

Recently, a retrospective analysis from the French Sarcoma Group ${ }^{36}$ on 1,513 patients with adult-type STS showed a statistically significant benefit in terms of five-years' overall survival (risk of death decreased by $13 \%$ ) and metastatis-free survival (metastatic risk decrease of $9 \%$ ), in patients with high-grade tumors.

Taken together, all these results suggest some role for chemotherapy, both in increasing local and distant control and thus improving outcome. This benefit is however limited and varies widely among the different histotypes. This could potentially be extrapolated also for RSTSS.

\section{Pre-operative Chemoradiation Therapy}

In extremity STS, the administration of pre-operative chemotherapy and RT was associated to a better outcome in a large retrospective analysis. ${ }^{37}$ Patients were divided into four five-year groups according to the time of their first operation. Sarcoma-specific mortality and local recurrence were significantly reduced in the last period dropping from 23 and $15 \%$ to 9 and $6 \%$ respectively. Furthermore, distant metastasis-free survival and the survival of patients with metastatic disease was improved. All the improvements were statistically significant, with similar proportions of patients with deep and high-grade tumors in all time periods. It is hypothesized that these improvements are related to the introduction in the latter cohorts of pre-operative combined chemoradiation therapy, practically never employed in the first three periods.

So far the same cannot be said in RSTSs. Pisters et al. enrolled 35 patients with primary or recurrent resectable retroperitoneal sarcoma in a phase I trial. ${ }^{38}$ The study protocol included the concurrent administration of 
doxorubicin by continuous infusion and pre-operative radiation therapy with a total dose of $50.4 \mathrm{~Gy}$. After radiologic restaging, operable patients underwent surgery plus external beam IORT. Pre-operative treatment was completed in $89 \%$ of patients. Six patients were excluded from surgical procedures because of clinical and radiologic progression and 29 patients underwent surgery, with $90 \%$ of them receiving a macroscopic radical resection-22 patients received IORT.

Two patients required hospital admission during the treatment. Upper gastrointestinal bleeding due to gastritis occurred in two patients. Grade 3 or 4 neutropenia was found in three patients. Eighteen per cent of patients suffered grade 3 or 4 nausea-but no patients had major episodes of vomiting. In conclusion, the study demonstrated that re-operative combined chemoradiation therapy was feasible and well tolerated. Delivery of IORT after complete resectional surgery can be considered to be a relatively safe procedure with a low risk of intra- or post-operative complications. Cumulative toxicity was not much higher than is usually reported in isolated chemotherapy treatment for STS.

A randomized phase 3 multicenter study comparing neoadjuvant chemotherapy consisting in etoposide, ifosfamide, and doxorubicin alone or with regional hyperthermia for localized high-risk extremity STS, was published recently ${ }^{39}-341$ patients were enrolled. At a median follow-up of 34 months, the incidence of death or local relapse was much higher in the chemotherapy alone group. However, side effects such as grade $3 / 4$ leukopenia were more common in the combined therapy group. Mild or moderate pain and skin burn were described as the principal adverse events attributable to hyperthermia. Deaths related to treatment toxicity amounted to one in the chemotherapy alone group and two in the combined group. This trial concluded that regional hyperthermia increases the benefit of chemotherapy, improving the local control and the survival of patients with high-grade STS.

An Italian multicenter phase ॥ trial, developed by our center in collaboration with three other Italian cancer institutions, investigating pre-operative chemoradiation in localized RSTSs has been concluded recently. ${ }^{40}$ Patients received three cycles of high-dose continuous ifosfamide infusion $\left(14 \mathrm{~g} / \mathrm{m}^{2}\right)$ for 14 days. Radiotherapy was started at the onset of the second cycle and administered up to a total dose of 50.4 Gy in 28 fractions. Surgery was scheduled four to six weeks after the end of RT. An intra-operative boost was allowed when clinically indicated. The overall pre-operative treatment plan was completed in 61 patients. Chemotherapy was completed in 65 patients, while RT was carried out in 74 patients. A partial response was obtained in eight patients, while stable disease was observed in 62. Four patients had systemic progression before surgery and were not operated upon and 80 patients underwent surgery (macroscopically complete in 71). Intra-operative RT was delivered in 15 patients (median dose $12.0 \mathrm{~Gy}$ ). At a median follow-up of two years (IQ range =1-3.7), 20 and 15 patients developed local and distant recurrence after surgery, respectively, while 17 patients died of disease. Three-year local recurrence-free survival,
Figure 3: Study Design of a Phase III Trial Exploring the Effectiveness of Pre-operative Radiotherapy Alone

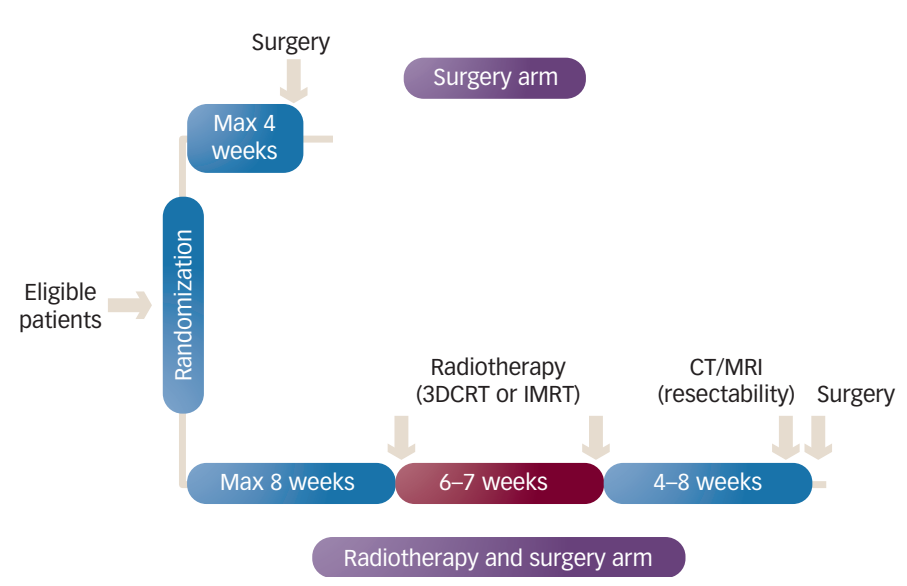

3DCRT $=$ three-dimensional conformational radiation therapy; $C T$ = computed tomography; $I M R T=$ Intensity Modulated Radiation Therapy; MRI = magnetic resonance imaging.

distant recurrence-free survival and overall survival were 68, 79 and $80 \%$, respectively. Long-term follow-up data are awaited. In conclusion, the combination of pre-operative high-dose long-infusion ifosfamide (HLI) and RT was feasible in two-thirds of patients, while pre-operative RT could be completed in the majority (74/84).

While systemic chemotherapy can be added to RT where this is felt to be appropriate in clinical practice in RSTSS, we lack a formal proof that this is of any benefit. This is why a phase III clinical trial exploring the role of RT alone was recently activated in Europe (see Figure 3).

\section{Conclusion}

The standard approach to RSTSS is based on wide surgical excision, involving multi-visceral resection, in order to obtain negative histologic margins and to decrease the risk of local recurrence. Despite aggressive surgical management, patients tend to relapse and multimodal treatment combining extended surgery and radiotherapy should be considered.

Despite the absence of randomized clinic trials, there is some retrospective evidence that RT plays a role in decreasing rates of local relapse, even if a significant advantage in terms of overall survival is not clearly reported. Neoadjuvant RT is generally preferred in order to avoid long-term complications such as bowel obstruction, fistulation, hydronephrosis, and neuropathic pain.

The role of chemotherapy remains controversial and chemotherapy is generally reserved for patients with metastatic or unresectable disease. Two recent clinical trials, however, have reported the safety and feasibility of neoadjuvant chemotherapy even when combined with RT. No further data are available regarding the benefit to patients in terms of disease-free and overall survival. The feasibility of such an approach deserves further research.
1. Mullinax J, Zager J, Gonzalez R, Current diagnosis and management of retroperitoneal sarcoma, Cancer Control, 2011;18:177-87.

2. Thomas D, O'Sullivan B, Gronchi A, Current concepts and future perspectives in retroperitoneal soft tissue sarcoma management, Expert Rev Anticancer Ther, 2009;9:1145-57.

3. Strauss D, Hayes A, Thomas J, Retroperitoneal tumours: review of management, Ann R Coll Surg Engl, 2011;93:275-80.
4. Lewis JJ, Leung D. Woodruff JM, Retroperitoneal soft tissue sarcoma: analysis of 500 patients treated and followed at a single institution, Cancer, 2004;100:2448-55.
5. Stoeckle $\mathrm{E}$, Coindre JM, Bonvalot $\mathrm{S}$, et al., Prognostic factors in


retroperitoneal sarcoma: a multivariate analysis of a series of 165 patients of the French Cancer Center federation Sarcoma Group, Cancer, 2001;92:359-68.

6. Ferrario $\mathrm{T}$, Karakousis $\mathrm{CP}$, Retroperitoneal sarcomas. Grade and survival, Arch Surg, 2003;138: 248-51.

7. Kilkenny JW, Bland KI, Copel EM. Retroperitoneal sarcoma: the university of Florida experience, J Am Coll Surg.

1996; 182:329-39.

8. Hassan I, Park SZ, Donohue JH, et al., Operative management of primary retroperitoneal sarcomas: a reappraisal of an institutional experience, Ann Surg, 2004;239:244-50.

9. Van Dalen T, Plooij JM, Van Coevorden F, et al., Long-term prognosis of primary retroperitoneal soft tissue sarcoma Eur J Surg Oncol, 2007:33:234-8.

10. Lehnert T, Cardona S, Hinz U, et al., Primary and locally recurrent retroperitoneal soft tissue sarcoma: local control and survival, Eur I Surg Oncol, 2009;35:986-93.

11. Strauss DC, Hayes AJ, Thway K, et al., Surgical management of primary retroperitoneal sarcoma, Br I Surg, 2010;101:520-3.

12. Gronchi A, Miceli R, Colombo $C$, et al., Frontline extended surgery is associated with improved survival in retroperitonea low- to intermediate-grade soft tissue sarcomas, Ann Oncol, 2012;23:1067-73.

13. Gronchi A, Bonvalot S, Le Cesne A, et al., Resection of uninvolved adjacent organs can be part of surgery for retroperitoneal soft tissue sarcoma, I Clin Oncol, 2009:27:2106-7.

14. Bonvalot $\mathrm{S}$, Rivoire $\mathrm{M}$, Castaing $\mathrm{M}$, et al., Primary retroperitoneal sarcomas: a multivariate analysis of surgical factors associated with local control, I Clin Oncol, 2009;27:31-7.

15. Bartlett $E$, Yoon $S$, Current treatment for the local control of retroperitoneal sarcomas, I Am Coll Surg, 2011; 213:436-46.

16. Bonvalot $\mathrm{S}$, Miceli $\mathrm{R}$, Berselli $\mathrm{M}$, et al., Aggressive surgery in retroperitoneal soft tissue sarcoma carried out at high volume centers is safe and is associated with improved local control, Ann Surg Oncol, 2010;17:1507-14.

17. Mendenhall W, Zlotecki R, Hochwald S, et al., Retroperitoneal soft tissue sarcoma, Cancer, 2005;104:669-75.

18. Katz $\mathrm{M}$, Choi $\mathrm{E}$, Pollock $\mathrm{R}$, Current concepts in multimodality therapy for retroperitoneal sarcoma, Expert Rev Anticancer Ther,
2007:7:159-68.

19. Pisters $P$, Harrison $L$, Leung $D$, et al., Long-term results of a prospective randomized trial of adjuvant brachytherapy in soft tissue sarcoma, I Clin Oncol, 1996;14:859-68.

20. Yang J, Chang A, Baker A, et al., Randomized prospective study of the benefit of adjuvant chemotherapy in the treatment of soft tissue sarcomas of the extremity, I Clin Oncol, 1998:16:197-203.

21. Sampath S, Hitchcock YJ, Shrieve DC, et al., Radiotherapy and extent of surgical resection in retroperitoneal soft tissue sarcoma: multi-institutional analysis of 261 patients, J surg Oncol, 2010;101:345-50.

22. Catton CN, O'Sullivan B, Kotwall C, et al., Outcome and prognosis in retroperitoneal soft tissue sarcoma, Int J Radiat Oncol Biol Phys, 1994;29:1005-10.

23. Gilbeau L, Kantor G, Stoeckle E, et al., Surgical resection and radiotherapy for primary retroperitoneal soft tissue sarcoma, Radiother Oncol, 2002;65:137-143.

24. Paumier A, Le Pechoux C, Beaudré A, et al., IMRT or conformal radiotherapy for adjuvant treatment of retroperitoneal sarcoma? Radiother Oncol, 2011; 99:73-8.

25. Jones JJ, Catton CN, O'Sullivan B, et al., Initial results of a trial of preoperative external-beam radiation therapy and postoperative brachytherapy for retroperitoneal sarcoma Ann Surg Oncol, 2002;9:346-54.

26. Sarcoma Meta-Analysis Collaboration, Adjuvant chemotherapy for localized resectable soft tissue sarcoma of adults: meta-analysis of individual data, Lancet, 1997;350:1647-54

27. Frustaci S, Gherlinzoni F, De Paoli A, et al., Adjuvant chemotherapy for adult soft tissue sarcomas of extremities and girdles: results of the Italian randomized cooperative trial, J Clin Oncol, 2001:19:1238-47.

28. Pervaiz N, Colterjohn N, Farrokhyar F, et al., A systematic meta-analysis of randomized controlled trials of adjuvant chemotherapy for localized resectable soft-tissue sarcoma Cancer, 2008:113:573-81

29. Gortzak E, Azzarelli A, Buesa J, et al., A randomized phase II study on neo-adjuvant chemotherapy for high-risk adult soft-tissue sarcoma, Eur I Cancer, 2001;37:1096-103.

30. Petrioli $\mathrm{R}$, Coratti A, Correale P, et al., Adjuvant epirubicin with or without ifosfamide for adult soft tissue sarcoma Ann I Clin. Oncol, 2002:25:468-73.

31. Woll PJ, Van Glabbeke M, Hoenberger P, et al., Adjuvan chemotherapy with doxorubicine and ifosfamide in resected soft tissue sarcoma; interim analysis of a phase III trial, I Clin Oncol, 2007:25:547.

32. Gronchi A, Frustaci $\mathrm{S}$, Mercuri $\mathrm{M}$, et al., Short, full-dose adjuvant chemotherapy in high-risk adult soft tissue sarcomas: a randomized clinical trial from the Italian Sarcoma Group and the Spanish Sarcoma Group, I Clin Oncol 2012;30:350-6.

33. Cormier JN, Huang $X$, Xing $Y$, et al., Cohort analysis of patients with localized, high-risk, extremity soft tissue sarcoma treated at two cancer centers: chemotherapy associated outcomes, Clin Oncol, 2004;22:4567-74.

34. Eilber FC, Eilber FR, Eckardt J, et al., The impact of chemotherapy on the survival of patients with high-grade primary extremity liposarcoma, Ann Surg, 2004;240:686-95

35. Ferrari A, Gronchi A, Casanova M, et al., Synovial sarcoma: a retrospective analysis of 271 patients of all ages treated at a single institution, Cancer, 2004:101:627-34.

36. Italiano A, Delva F, Mathoulin-Pellissier S, et al., Effect of adjuvant chemotherapy on survival in FNCLCC grade 3 sof tissue sarcoma: a multivariate analysis of the French Sarcoma Group database, Ann Onc, 2010;21:2436-41.

37. Gronchi A, Miceli R, Colombo C, et al., Primary extremity soft tissue sarcomas: outcome improvement over time at a single institution, Ann Oncol, 2011:22:1675-81.

38. Pisters W, Ballo $M$, Fenstermacher $M$, et al., Phase I trial of preoperative concurrent doxorubicin and radiation therapy, surgical resection and intraoperative electron-beam radiation therapy for patients with localized retroperitoneal sarcoma, I Clin Oncol, 2003:21:3092-7.

39. Issels R, Lindner L, Verweij $\rfloor$, et al., Neo adjuvant chemotherapy alone or with regional hyperthermia for localised high-risk soft tissue sarcoma: a randomised phase 3 multicentre study, Lancet Oncol, 2010:11:561-70.

40. Gronchi A, De Paoli, Dani C, et al., Preoperative chemo-radiation therapy for localized retroperitoneal soft tissue sarcoma (STS): phase II study from the Italian Sarcoma Group, I Clin Oncol, 2011;29:10020. 